\title{
Ferromagnetic and spin wave resonances in thin layer of expanded austenite phase
}

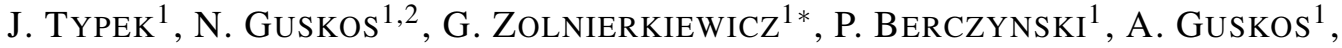 \\ J. BARANOWSKA ${ }^{3}$, S. FRYSKA ${ }^{3}$ \\ ${ }^{1}$ Institute of Physics, West Pomeranian University of Technology, al. Piastów 48, 70-311 Szczecin, Poland \\ ${ }^{2}$ Department of Solid State, Faculty of Physics, University of Athens, Panepistimiopolis, 15784 , Greece \\ ${ }^{3}$ Institute of Materials Science and Engineering, West Pomeranian University of Technology, \\ al. Piastów 17, 70-310 Szczecin, Poland
}

\begin{abstract}
Four samples of austenite coatings deposited by reactive magnetron sputtering on silicon substrate at four different temperatures and pressures were investigated by ferromagnetic resonance (FMR) method at room temperature. The expanded austenite phase $\mathrm{S}\left(\gamma_{N}\right)$ layers with thickness in the $160-273 \mathrm{~nm}$ range and concentration of magnetic atoms: $72 \% \mathrm{Fe}, 18 \%$ $\mathrm{Cr}$ and $10 \% \mathrm{Ni}$, were obtained. The coatings with nanometric size grains were strongly textured and grown mostly in [100] direction, perpendicular to the sample surface. Intense FMR spectra were recorded at various angles between the static magnetic field direction and the sample surface. A strong magnetic anisotropy of the main uniform FMR mode was observed and the effective magnetization $4 \pi M_{e f f}$ determined. Spin wave resonance (SWR) modes were observed in all investigated samples in out-of-plane geometry of the magnetic field. The resonance fields of SWR modes in our samples varied linearly with the spin wave mode number. The value of the effective magnon stiffness constant was determined assuming a parabolic shape of the magnetization variation across the sample thickness.
\end{abstract}

Keywords: expanded austenite; thin layer; ferromagnetic resonance; spin waves

(C) Wroclaw University of Technology.

\section{Introduction}

The austenite stainless steels play a very important role in various technological applications and are intensively studied both experimentally and theoretically, e.g. [1-7]. They are largely used in corrosive industrial environment, such as in the chemical and food industries. These steels have excellent corrosion resistance due to formation of a thin surface layer of oxide, but their wear and hardness are relatively poor. Surface modifications techniques such as nitriding or layers deposition have been used to increase their surface hardness and the wear resistance. Especially the so-called low temperature nitriding is a very useful method to increase the hardness and wear resistance of the austenitic stainless steel [8-10]. When nitriding is carried out in temperature below $450{ }^{\circ} \mathrm{C}$, a

*E-mail: grzegorz.zolnierkiewicz@ zut.edu.pl nitrogen-expanded austenite layer with high hardness and good corrosion resistance is obtained [1114]. In this metastable phase, denoted as $\gamma_{N}$ or $S$ phase, nitrogen remains in a solid solution. This phase has fcc structure but with much larger lattice parameter than austenite. One of the characteristic features of this phase are XRD reflections shifted to lower Bragg angles with respect to those for typical austenite and the shift increases along with increasing nitrogen content in S-phase [15].

Magnetic properties and magnetic effects play an important role in materials with austenite phases in which the spin wave excitations are often observed, e.g. [16-21]. Ferromagnetic resonance (FMR) is one of the most powerful techniques for the study of magnetic nanoparticles [22-25]. FMR and its generalization for non-uniform excitations known as spin wave resonance (SWR) are very useful methods for investigation of magnetic anisotropy, magnetic interactions and identification 
of diverse phases in layered samples of stainless steel. It was demonstrated by MFM and Mössbauer studies that in contrast to austenitic substrate the S-phase has magnetic properties [26-28]. Nitrided layers obtained on austenitic stainless steel by diffusive methods have a gradient nitrogen composition - the nitrogen content is the highest on the surface and gradually decreases towards the substrate [29]. Due to this fact it is difficult to correlate the physical properties of the layer with nitrogen content or microstructure. Coatings composed of S-phase can be also obtained by means of physical methods e.g. using reactive magnetron deposition [30-33]. Such coatings have homogenous nitrogen content which makes their characterization easier.

The aim of this work was the preparation of four samples of the expanded austenite phase obtained at different pressures and temperatures, by means of reactive magnetron deposition and the investigation of their magnetic properties by using magnetic resonance method at microwave frequency. It is expected that the spin wave excitations effect can significantly modify the FMR spectrum and the proposed method could be very useful, providing useful magnetic characteristics of materials with austenite phase.

\section{Experimental}

The coatings were deposited by reactive sputter deposition at different temperatures and total gas pressures. The deposition chamber was a cylinder with $300 \mathrm{~mm}$ diameter and $400 \mathrm{~mm}$ height. The magnetron gun with the target was located on the bottom of the chamber. The $50 \mathrm{~mm}$ diameter target was made of X10CrNi18-10 stainless steel. The target was powdered using a $750 \mathrm{~W}$ and $20 \mathrm{kHz}$ pulsed dc supply at a mean target power of $200 \mathrm{~W}$ ( $\sim 0.3 \mathrm{~A})$. The chamber was pumped down by a turbo pump to a base pressure of about $10^{-5} \mathrm{~Pa}$. The pressure was measured by means of MKS Barotron (capacitance manometer) gauge, and the inlet gas mixture $\left(\mathrm{Ar} / \mathrm{N}_{2}=10 / 3\right)$ was controlled by MKS mass-flow controller. Silicon plates used as substrates were ultrasonically degreased, rinsed in alcohol and dried in hot air. Then, the substrates were placed on a heating holder and biased with RF source (power of $2 \mathrm{~W}$ ). The coatings were deposited for $90 \mathrm{~min}$ on the silicon substrates. Before the deposition, the substrates were ion cleaned at a pressure of $2.66 \mathrm{~Pa}$ for $10 \mathrm{~min}$. Samples designations and the parameters of deposition are shown in Table 1.

Table 1. Sample designation, parameters of thin films depositions, and thickness of obtained coatings.

\begin{tabular}{cccc}
\hline $\begin{array}{c}\text { Sample } \\
\text { designation }\end{array}$ & $\begin{array}{c}\text { Temperature Pressure Thickness } \\
{\left[{ }^{\circ} \mathrm{C}\right]}\end{array}$ & $\begin{array}{c}{[\mathrm{Pa}]} \\
{[\mathrm{nm}]}\end{array}$ \\
\hline \hline 1 & 165 & 0.40 & 193 \\
2 & 284 & 0.27 & 160 \\
3 & 367 & 0.27 & 273 \\
4 & 367 & 0.53 & 247 \\
\hline
\end{tabular}

To characterize the coatings X-ray diffraction (XRD), atomic force microscope (AFM) and electron probe microanalysis (EPMS EDS) methods were used. XRD (from X'pert Panalytical) with Bragg-Brentano geometry for the range of 2 theta angles between 38 and $67^{\circ}$ and $\mathrm{CuK} \alpha$ radiations was applied. AFM (from Nanoscope IV, Veeco) was used to investigate surface morphology. EPMS EDS (from Noran Instruments IBEX) with accelerating voltage of $5 \mathrm{kV}$ and beam current of $15 \mathrm{nA}$ was used to estimate the elemental composition of the coatings. The following concentrations of magnetic atoms in our samples were found: $72 \% \mathrm{Fe}$, $18 \% \mathrm{Cr}$ and $10 \% \mathrm{Ni}$.

The FMR measurements at room temperature (RT) were carried out using Bruker E 500 spectrometer working in the X-band $(v=9.4 \mathrm{GHz})$ of microwave absorption and using $100 \mathrm{kHz}$ magnetic field modulation The investigated samples (rectangular plates $5 \times 1 \mathrm{~mm}$ ) were placed in the resonance cavity on an insert enabling rotation along vertical axis (Fig. 1). The applied dc magnetic field was confined to the horizontal plane, and a weak microwave field acting on the sample was vertical. The direction of the external magnetic field with respect to the sample surface was characterized by angle $\theta$ (Fig. 1) and could be changed in the 
$0-180^{\circ}$ range. By rotation of the sample rod, the applied static magnetic field could be aligned in $H_{\|}$(in-plane, for $\theta=0^{\circ}$ ) and $H_{\perp}$ (out-of-plain, for $\theta=90^{\circ}$ ) geometries. As usual, the registered FMR spectra were in the form of the first derivative of the absorption as a function of the applied magnetic field.

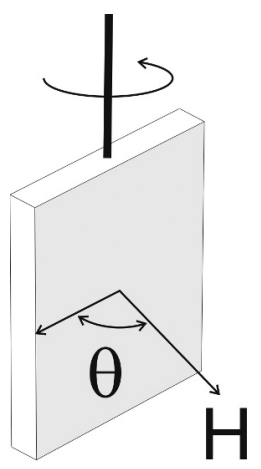

Fig. 1. Sketch of investigated sample on an insert enabling rotation along vertical axis making an angle $\theta$ with the direction of the external magnetic field.

\section{Results and discussion}

In the XRD patterns (Fig. 2), for S-phase only, the peaks originating from (100) plains are visible. It demonstrates that the layers are strongly textured and the grains grow in [100] directions perpendicular to the samples surface. It can be observed that a change in total pressure has an influence on Sphase peak position. An increase in total pressure and thereby increase in nitrogen partial pressure causes a shift of S-phase peaks towards smaller 2 theta angles. It is well correlated with nitrogen content. Thorough investigation of XRD patterns it has been shown that at the highest temperatures of formation (samples 3 and 4), beside the S-phase, an unknown second phase is found (Fig. 2), however, its origin has been unknown so far. Moreover, in sample 1 the presence of grains grown in [111] direction perpendicular to the sample surface is visible. Grains in all coatings are nanometric in size as revealed by AFM study of the surfaces.

In Fig. 3 the magnetic resonance spectra of all four samples in the parallel configuration (exter-

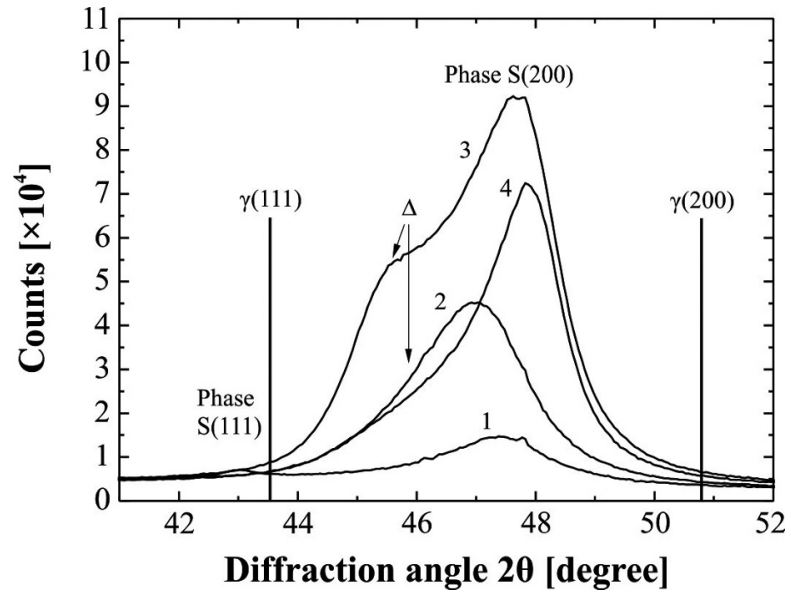

Fig. 2. XRD patterns of investigated samples; $\gamma$-stainless steel with [111] or [200] orientation, Sphase with [111] or [200] orientation, $\Delta-$ unknown phase.

nal magnetic field parallel to the sample surface) are presented. The spectra are presented in a usual form of a derivative of the absorption as a function of applied magnetic field. The FMR spectrum consists of only one peak with non-symmetrical shape, centered at a magnetic field of about $1 \mathrm{kG}$. This peak is often called uniform or FMR mode. The shape of the FMR peak indicates the presence of at least two components: one stronger, placed on the low-field side and the other weaker, on the high-field side. The different shapes of the FMR lines registered in four different samples might be due to an inherent in-plane magnetic anisotropy. Assuming a random distribution of in-plane magnetic axes of the nanograins in the sample surface plane, the resulting FMR spectrum with two components could be thought as manifestation of magnetic anisotropy existing in that plane. In that case the difference in the resonance fields of the two components would be a direct measure of the in-plane magnetic anisotropy. For samples 1, 2, 3, and 4 that difference is $127,194,168$, and $209 \mathrm{G}$, respectively.

As the magnetic field is rotated away from the in-plane toward out-of-the-plane configuration, the uniform mode resonance line shifts towards higher magnetic fields. In Fig. 4 - 7, the magnetic resonance spectra of samples $1-4$, taken at different 


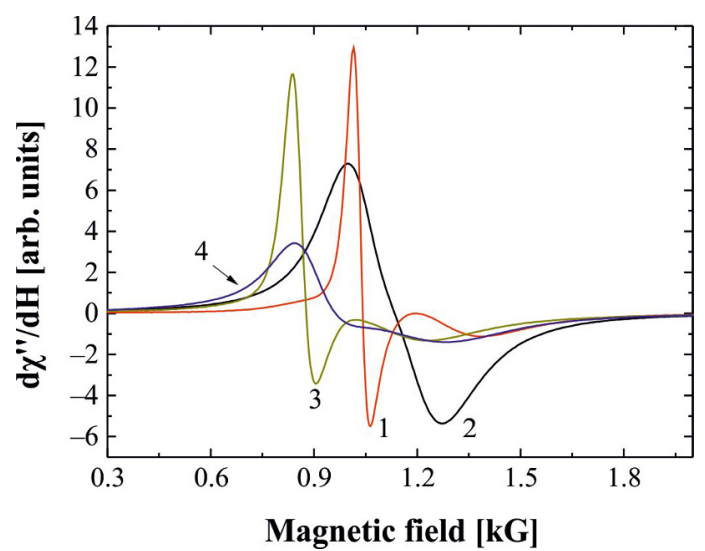

Fig. 3. The FMR lines of samples $1-4$ registered at $\theta=0^{\circ}$, corresponding to in-plane orientation of the magnetic field.

angles $\theta$ of an external magnetic field relative to the sample surface, are presented. It can be seen that when the external magnetic field is close to the normal to the layer surface $\left(\theta \sim 90^{\circ}\right)$ additional resonance lines in the magnetic spectrum appear. They are identified as the spin wave resonances and will be discussed latter. In Fig. 6 (sample 3) an additional resonance line is observed near magnetic field $\sim 1 \mathrm{kG}$ which does not shows significant anisotropy. This line could be attributed to the presence of an extra phase observed in XRD experiment which was most visible in that sample. At $\theta=$ $90^{\circ}$ the FMR line is in a very high field, and in case of samples 1,3 , and 4 it is even beyond the range of our spectrometer $\left(H_{\max }=14 \mathrm{kG}\right)$. Therefore, only the FMR spectrum of sample 2 could be discussed more thoroughly.

In Fig. 8 an angular dependence of the resonance field of the FMR mode for four investigated samples is presented. In case of an FMR line, the formulas for the resonance fields in parallel $\left(H_{\|}\right)$ and perpendicular $\left(H_{\perp}\right)$ configurations of a thin magnetic film have been derived by Kittel [34]. They are given by the equations:

$$
\begin{gathered}
h v=g_{e f f} \mu_{B}\left(H_{\perp}-4 \pi M_{e f f}\right) \\
h v=g_{e f f} \mu_{B} \sqrt{\left[H_{\|}\left(H_{\|}+4 \pi M_{e f f}\right)\right]}
\end{gathered}
$$

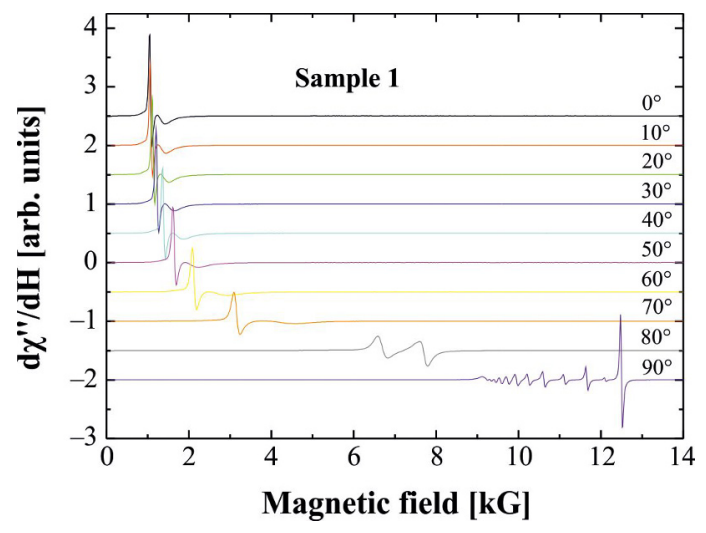

Fig. 4. Magnetic resonance spectra of sample 1 taken at various angles $\theta$ of the external magnetic field relative to the sample surface.

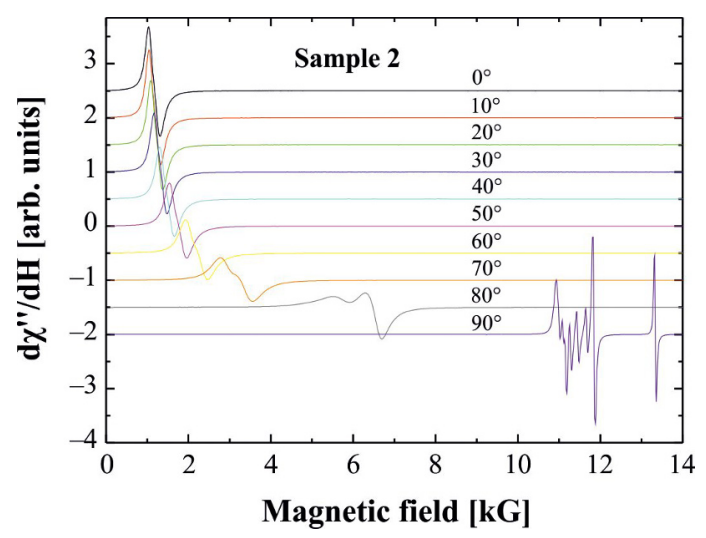

Fig. 5. Magnetic resonance spectra of sample 2 taken at various orientations $\theta$ of the external magnetic field relative to the sample surface.

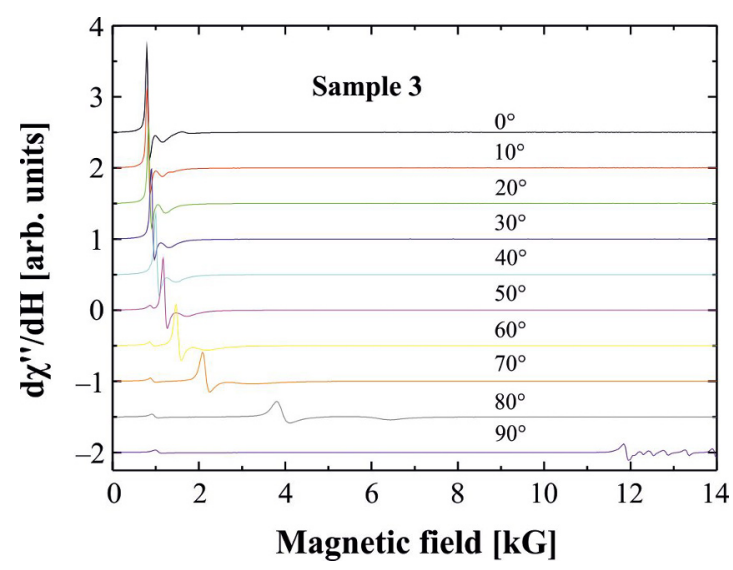

Fig. 6. Magnetic resonance spectra of sample 3 taken at various orientations $\theta$ of the external magnetic field relative to the sample surface. 


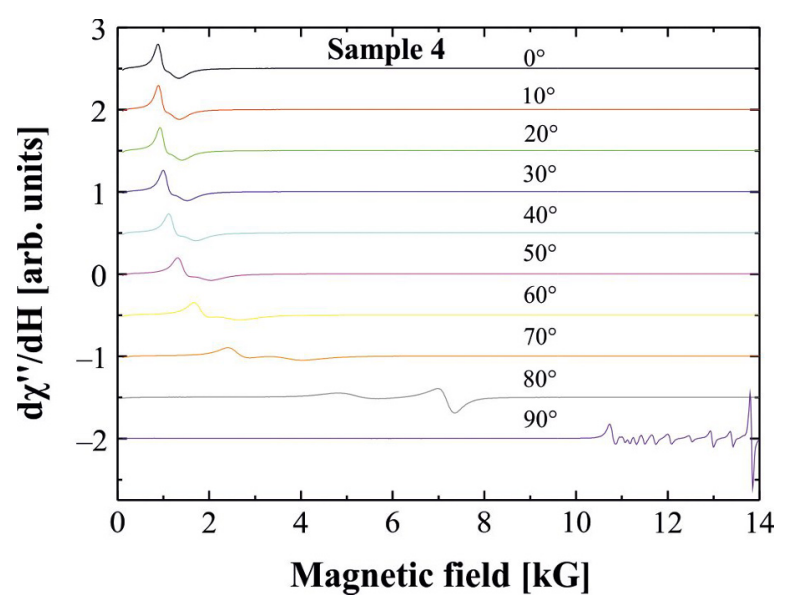

Fig. 7. Magnetic resonance spectra of sample 4 taken at various orientations $\theta$ of the external magnetic field relative to the sample surface.

where Eq. (1) corresponds to the perpendicular and Eq. (2) to the parallel orientation of the external magnetic field. In the above equations $v$ is the resonance frequency, $\mu_{B}$ is the Bohr magneton, $H_{\perp}$ and $H_{\|}$are the experimental values of magnetic resonance fields, $g_{\text {eff }}$ is the effective spectroscopic $g$-factor and $4 \pi M_{\text {eff }}$ is the effective magnetization. Using the Kittel equations for sample 2, the following values were obtained: $g_{\text {eff }}=1.87,4 \pi M_{\text {eff }}=9.74 \mathrm{kG}$. For a pure iron film $4 \pi M_{\text {eff }}=21.5 \mathrm{kG}$. The low value of effective magnetization for sample 2 may result from the low value of the filling factor and the action of the demagnetization factor due to textured structure of our layer [35]. Although for the other three samples an accurate calculation of $4 \pi M_{\text {eff }}$ is not possible (the FMR mode lies above $14 \mathrm{kG}$ ), it is still possible to estimate its value by inspecting the structure of the high-field lines visible in each sample (Fig. 4 -7). The value of effective magnetization is proportional to the spread of the spectra and this separation is easily visible in the following sequence: sample $2<$ sample $1<$ sample $4<$ sample 3. This succession is exactly the same as the sequence of increasing sample thickness. Thus the thicker the sample, the bigger the value of the effective magnetization. That dependence is easy to recognize because in thicker samples the proportion of surface spins is smaller and the magnetic moment

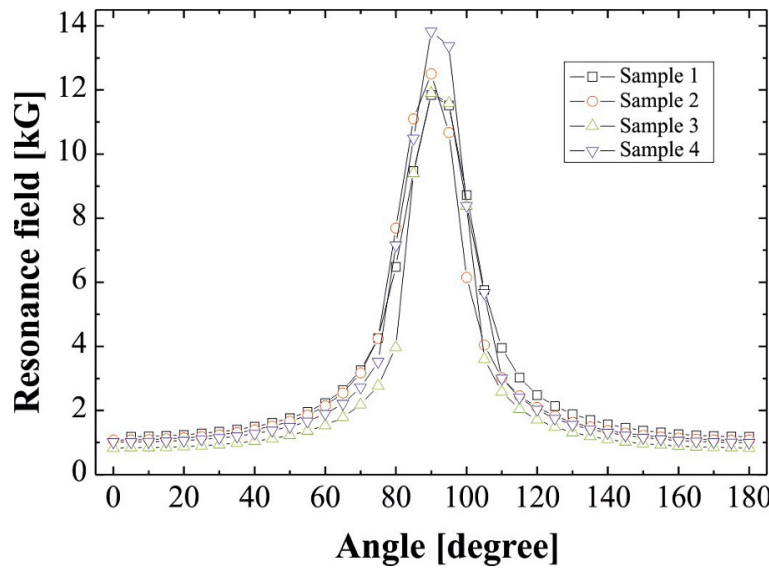

Fig. 8. Angular dependence of the resonance field of the FMR uniform mode for four investigated samples.

of the surface spins can be smaller in comparison with the spins located in the bulk of a coating.

The FMR resonance mode discussed above corresponds to the spatially uniform precession of magnetization. In the language of spin waves this type of mode can be regarded as a spin wave with the wave vector $\mathbf{k}=0$ [36]. In thin films, certain magnons, depending on the boundary condition of the film, could be amplified and observed as additional absorption peaks. They are referred as spin wave resonances and are clearly visible in our spectra at angles close to $\theta \sim 90^{\circ}$. The SWR spectrum consists of a main resonance line located at the highest field with a series of weaker satellite peaks at lower fields. Assuming that the spin precession of the spin waves at the surface is frozen (surface spins are pinned) the following equation for the resonance field $H_{n}$ could be obtained [37]:

$$
H_{n}=4 \pi M_{e f f}+\frac{\omega}{\gamma}-D_{e f f} \frac{k_{n}^{2}}{\gamma \hbar}
$$

with $\gamma=\frac{g \mu_{B}}{\hbar}, k_{n}=\frac{n \pi}{L}$, where $4 \pi M_{\text {eff }}$ is the effective magnetization, $\omega$ is the microwave frequency, $\gamma$ is the gyromagnetic ratio, $D_{e f f}$ is the effective magnon stiffness constant, $k_{n}$ is the wave number of the $n$-th spin wave mode, $\mu_{B}$ is Bohr magneton, $L$ is the thickness of the layer and $\hbar$ is Planck constant. In Eq. (3), valid for the so called Kittel boundary conditions, $n$ is an odd integer. The effective magnon stiffness constant gives a mea- 
sure of the strength of exchange interaction that tries to keep magnetic moments parallel. It could be calculated by considering two different spin-wave modes. The integer $n$ corresponds to the number of half wavelengths of spin wave spectra within the thickness of the film.

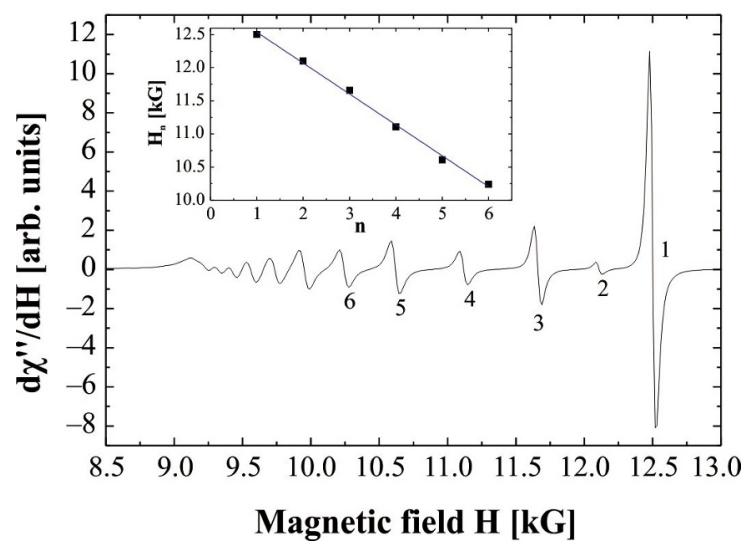

Fig. 9. SWR spectra for sample 1 in the out-of-plain orientation $\left(\theta=90^{\circ}\right)$ of the magnetic field. The inset shows the dependence of $H_{n}$ on the spin wave number $n$.

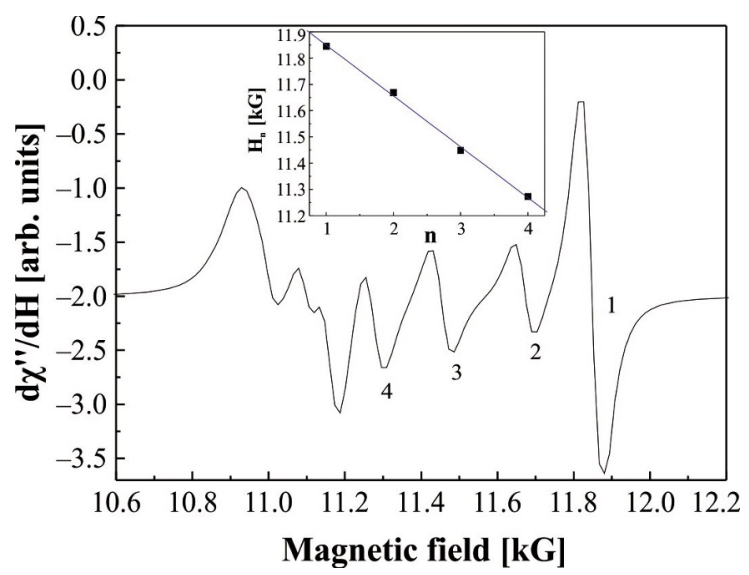

Fig. 10. SWR spectra for sample 2 in the out-of-plain orientation $\left(\theta=90^{\circ}\right)$ of the magnetic field. The inset shows the dependence of $H_{n}$ on the spin wave number $n$.

In Fig. 9 - 11 the SWR spectra for samples 1, 2 and 4 in the out-of-plain orientation $\left(\theta=90^{\circ}\right)$ of the magnetic field are presented. The insets show the dependence of $H_{n}$ on the spin wave number $n$.

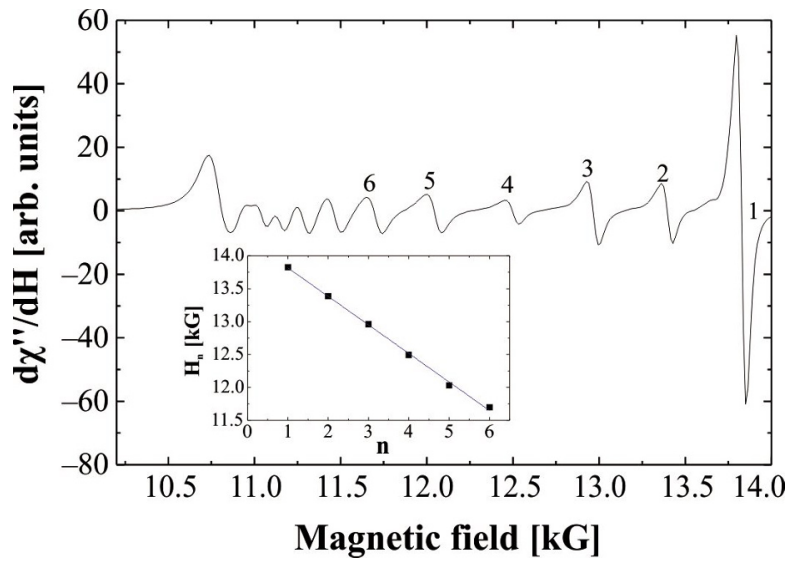

Fig. 11. SWR spectra for sample 4 in the out-of-plain orientation $\left(\theta=90^{\circ}\right)$ of the magnetic field. The inset shows the dependence of $H_{n}$ on the spin wave number $n$.

Surprisingly, the resonance fields of SWR modes in these samples vary linearly with $n$. Such dependence has been seen previously in $\mathrm{Ga}_{1-x} \mathrm{Mn}_{x} \mathrm{As}$ thin films [38-40]. The origin of the anomalous spin wave dispersion has been attributed to the dependence of magnetic parameters inside the film on the distance from the surface. An inhomogeneity in the profile of either magnetization, spin stiffness, or magnetic anisotropy could explain the observed anomaly. The experimental and theoretical analysis indicate that variation of the magnetic anisotropy with a linear [38] or a quadratic dependence [40] on the distance $z$ from the surface can explain the obtained results. In the following we will apply the method proposed by Liu X. et al. [40]. Assuming that the observed linear dependence of resonance fields on $n$ is the result of symmetrical parabolic form of $4 \pi M_{e f f}$ along $z$ axis $(-L / 2 \leqslant z \leqslant L / 2)$ :

$$
4 \pi M_{e f f}(z)=4 \pi M_{e f f}^{0}\left(1-\frac{4 \varepsilon z^{2}}{g \mu_{B}}\right)
$$

where $\varepsilon$ is the magnetic distortion parameter used to define the profile of magnetic anisotropy along $z$. The $n$-th ( $n$ is an odd integer) SWR mode occurs at magnetic field $H_{n}$ given by:

$$
H_{n}=H_{0}-\left(n-\frac{1}{2}\right)\left(\frac{4}{L}\right) \sqrt{4 \pi M_{e f f}^{o} \varepsilon \frac{D_{e f f}}{g \mu_{B}}}
$$


As the value of $\varepsilon$ is consistent with the field separation between the highest and the lowest observed SWR modes, for sample 1 we obtain $\varepsilon=\frac{\Delta H}{4 \pi M_{\text {eff }}}=\frac{848 G}{9740 G}=0.087$. From Eq. (5) the following value of the effective exchange stiffness constant $D_{e f f}=80.1 \mathrm{meV} \cdot \AA^{2}$ is calculated. This value is two times larger than the one obtained for (Ga, Mn)As films [39] but it is only about one-third value measured for bulk $\mathrm{Fe}\left(280 \mathrm{meV} \cdot \AA^{2}\right)$ [41].

\section{Conclusions}

Four samples of magnetic layers of the expanded austenite phase were obtained at different pressures and temperatures on silicon. The intention was to produce, at various process parameters, the $\mathrm{S}$ phase coatings with a similar texture to avoid the possible influence of the texture on magnetic behavior of S-phase. All S-phase coatings were obtained by reactive magnetron sputtering and had a texture with (100) plains parallel to the coating surface. Therefore it was necessary to choose a combination of temperature and pressure values to achieve the intended purpose. Selection of these parameters was done basing on previous experience presented by Baranowska J. et al. [33]. Furthermore, the chosen parameters allowed us to obtain the coatings with the phase structure as shown in Fig. 2. Moreover, this figure shows the presence of an additional phase $\Delta$, whose intensity also depends on the parameters used. The present paper aimed at finding correlations between the parameters of the obtained layers (such as layer thickness, texture, and phase structure) and magnetic characteristics determined by magnetic resonance spectroscopy. The presence of narrow resonance FMR lines indicated a highly homogenous texture with (100) plains parallel to the coating surface. It was found that the additional $\Delta$ phase, registered in the layers formed at high temperature, produced a magnetic resonance line in a low magnetic field. It was deduced that the value of the effective magnetization scales with the film thickness indicating a smaller magnetic moment of surface spins. The presence of even and odd spin wave modes in SWR spectra indicated that the pinning was asymmetric at the surface and interface of the films. Assuming sym- metrical parabolic form of $4 \pi M_{\text {eff }}$ across sample thickness, the value of the effective exchange stiffness constant $D_{e f f}=80.1 \mathrm{meV} \cdot \AA^{2}$ was calculated.

\section{References}

[1] Colling D.A., J. Appl. Phys., 41 (1970), 1038.

[2] Jiang D.E., Carter E.A., Phys. Rev. B, 67 (2003), 214103.

[3] Garcia-Mateo C., Caballero F.G., Mater. Trans., 46 (2005), 1839.

[4] Zhong N., Wang X., Rong Y., Wang L., J. Mater. Sci. Technol., 22 (2006), 751.

[5] Rajasekhara S., Ferreira P.J., Acta Mater., 59 (2011), 738.

[6] Dmitrieva O., Ponge D., Inden G., Millan J., Choi P., Sietsma J., RaAbe D., Acta Mater, 59 (2011), 364.

[7] CApò SÀnchez J., Huallpa E., FARina P., Padovese L.R., Goldenstein H., J. Appl. Phys., 110 (2011), 083916.

[8] Blawert C., Mordike B.L., Surf. Coat. Tech., 116 119 (1999), 352.

[9] LeI M.K., ZHU X.M., Surf. Coat. Tech., 193 (2005), 22.

[10] Baranowska J., Franklin S.E., Kochmańska A., Wear, 263 (2007), 669.

[11] Baranowska J., Franklin S.E., Pelletier C.G.N., Wear, 259 (2005), 432.

[12] Baranowska J., Arnold B., Surf. Coat. Tech., 200 (2006), 6623.

[13] Zhang Z.L., Bell T., Surf. Eng., 1 (1985), 131.

[14] Guermmaz M., Mosser A., Grob J.J., Stuck R., Surf. Coat. Tech., 100 - 101 (1998), 353.

[15] Christiansen T., Somers M.A.J., Scripta Mater., 50 (2004), 35.

[16] Stringfellow M.W., J. Phys. C Solid State, 1 (1968), 950.

[17] Hoekstra B., VAn Stapele R.P., Robertson J.M., J. Appl. Phys., 48 (1977), 382.

[18] Morais P.C., Tronconi A.L., Neto K. S., J. Appl. Phys., 55 (1984), 3744.

[19] Pajda M., KudrnovskÝ J., Turek I., Drchal V., Bruno P., Phys. Rev. B, 64 (2001), 174402.

[20] Ekholm M., Zapolsky H., Ruban A.V., Vernyhora I., Ledue D., Abrikosov I.A., Phys. Rev. Lett., 105 (2010), 167208.

[21] Bergman A., Skubic B., Hellsvik J., NordStröm L., Delin A., ERIKSSOn O., Phys. Rev. B, 83 (2011), 224429.

[22] Bodziony T., Guskos N., Typek J., Roslaniec Z., Narkiewicz U., KWiatKowsKa M., MaryNIAK M., Mater. Sci.-Poland, 23 (2005), 1055.

[23] Guskos N., TyPek J., Maryniak M., NARKiEWiCZ U., Kucharewicz I., Wróbel R., Mater. Sci.Poland, 23 (2005), 1001. 
[24] Guskos N., Typek J., Maryniak M., ŻoŁnierkiewicz G., PodsiadŁy M., Arabczyk W., LENDZION-BIEluń Z., NARKIEWICZ U., Mater. Sci.-Poland, 24 (2006), 1095.

[25] Guskos N., TypeK J., ŻoŁnierkiewicz G., WARdal K., Guskos A., Berczyński P., PETRidis D., Mater. Sci.-Poland, 31 (4) (2013), 587.

[26] Baranowska J., Surf. Coat. Tech.,180 - 181 (2004), 145.

[27] El-Hossary F.M., Surf. Eng., 16 (2000), 491.

[28] Jirásková Y., SVOboda M., SchneEweiss O., Žá́ T., Czech. J. Phys., 52 (2002), A61.

[29] Baranowska J., Defect Diffus. Forum, 312 - 315 (2011), 530.

[30] Bourjot A., Foos M., Frantz C., Surf. Coat. Tech., $44-45$ (1990), 533.

[31] Kappaganthu S.R., Sun Y., Surf. Coat. Tech., 198 (2005), 59.

[32] Saker A., He H., Czerwiec T., Li X., Tran HuU L., Dong C., Michel H. Frantz C., Thin Solid Films, 516 (2008), 1029.

[33] Baranowska J., Fryska S., Suszko T., Vacuum, 90 (2013), 160.

[34] Kitell C., Phys. Rev., 73 (1948), 155.
[35] Rubinstein M., Tejada J., Zhang X.X., J. Appl. Phys., 75 (1994), 6557.

[36] LiU X., Furdyna J., J. Phys.-Condens. Mat., 18 (2006), R245.

[37] Pandey H., Joshi P.C., Pant R.P., Prasad R., Auluck S., Budhani R.C., J. Appl. Phys., 111 (2012), 023912.

[38] Goennenwein S.T.B., Graf T., Wassner T., BRAndt M.S., Stutzmann M., PhilipP J.B., Gross R., Krieger M., Zurn K., Ziemann P., Koeder A., Frank S., Schoch W., WaAg A., Appl. Phys. Lett., 82 (2003), 730.

[39] RAPPOPORT T.G., REDLINSKI P., LIU X., ZARAND G., FURDYNA J.K., JANKO B., Phys. Rev. B, 69 (2004), 125213.

[40] Liu X., Zhou Y.Y., Furdyna J.K., Phys. Rev. B, 75 (2007), 195220.

[41] Mоoк H.A., Nicklow R.M., Phys. Rev. B, 7 (1973), 336.
Received 2013-12-23

Accepted 2014-03-15 